\title{
Porocarcinoma écrino: relato de quatro casos e revisão da literatura
}

\author{
Eccrine porocarcinoma: report of four cases and literature review
}

\author{
Fabiane Andrade Mulinari-Brenner ${ }^{1}$ \\ Carlos Augusto Silva Bastos ${ }^{3}$ \\ Jesus Rodriguez Santamaria
}

\author{
Maira Mitsue Mukai ${ }^{2}$ \\ Ezio Augusto Amaral Filho ${ }^{4}$ \\ José Fillus Neto ${ }^{6}$
}

\begin{abstract}
Resumo: Porocarcinoma écrino é um tumor de pele raro derivado do acrossiríngeo. Quatro casos de porocarcinoma écrino são estudados. Os pacientes tinham idade entre 49 e 64 anos, sendo dois homens e duas mulheres. O tempo de crescimento dos tumores variou de um a 20 anos. As lesões mediam de $1,2 \times 2$ a $4 \times 5 \mathrm{~cm}$ e se localizavam nas extremidades ou na face. Dois pacientes apresentavam carcinoma basocelular em sítios diferentes do porocarcinoma écrino. Duas das biópsias foram previamente descritas como poroma écrino; entretanto, malignidade foi observada em todos os casos após excisão ampla. Portanto, em toda biópsia de pele compatível com poroma écrino, deve-se considerar a possibilidade de malignidade.

Palavras-chave: Acrospiroma écrino; Adenocarcinoma; Carcinoma; Glândulas

Abstract: Eccrine porocarcinoma is an extremely rare skin tumor derived from the acrosyringium. Four cases of eccrine porocarcinoma are described, two males and two females, aged from 49 to 64 years. Tumors grew in an interval of one to 20 years. Lesions ranged from $1.2 \times 2 \mathrm{~cm}$ to $4 \times 5 \mathrm{~cm}$ and were on limbs or face. Two patients had basal cell carcinoma in a site different from the eccrine porocarcinoma. Two biopsies were described as eccrine poroma. Malignancy was observed in all cases after wide excision. Therefore, eccrine porocarcinoma should always be considered after a biopsy compatible with eccrine poroma.

Keywords: Acrospiroma, eccrine; Adenocarcinoma; Carcinoma; Eccrine glands
\end{abstract}

\section{INTRODUÇÃO}

Os carcinomas das glândulas sudoríparas são raros, compreendendo cerca de $0,005 \%$ de todas as neoplasias da pele. ${ }^{1} \mathrm{O}$ porocarcinoma écrino (PCE) ou poroma écrino maligno é um tumor de pele extremamente raro, embora seja relativamente comum ao se considerarem os carcinomas de glândulas sudorí- paras. Surge da porção intraepitelial da unidade da glândula sudorípara écrina, o acrossiríngio, podendo apresentar-se como tumor primário ou, mais comumente, como uma transformação maligna de um poroma écrino (PE). ${ }^{2}$ A primeira descrição do tumor foi em 1963 por Pinkus e Mehregan. ${ }^{3}$

\footnotetext{
Recebido em 04.10.2004.

Aprovado pelo Conselho Consultivo e aceito para publicação em 26.06.09

* Trabalho realizado no Serviço de Dermatologia, Departamento de Medicina Interna e Departamento de Patologia, Hospital de Clínicas, Universidade Federal do Paraná (UFPR) - Curitiba, Brasil.

Conflito de interesse: Nenhum / Conflict of interest: None

Suporte financeiro: Nenhum / Financial funding: None

1 Dermatologista, professora assistente de dermatologia da Universidade Federal do Paraná, chefe do Serviço de Dermatologia da Universidade Federal do Paraná (UFPR) - Curitiba (PR), Brasil.

Dermatologista, professora substituta de dermatologia da Universidade Federal do Paraná (UFPR) - Curitiba (PR), Brasil.

3 Dermatologista, preceptor do ambulatório do Hospital de Clínicas, Universidade Federal do Paraná, e do Centro de Dermatologia e Doenças Infecciosas Souza Araújo - Curitiba (PR), Brasil.

Cirurgião oncológico do Centro de Dermatologia e Doenças Infecciosas Souza Araújo, mestre em cirurgia pela Universidade Federal do Paraná (UFPR) Curitiba (PR), Brasil.

Dermatologista, professor assistente de dermatologia da Universidade Federal do Paraná (UFPR) - Curitiba (PR), Brasil.

Professor adjunto do Departamento de Patologia Médica do Hospital de Clínicas de Curitiba - Curitiba (PR), Brasil.

(C)2009 by Anais Brasileiros de Dermatologia
} 


\section{RELATO DOS CASOS}

Foram revisados detalhadamente os achados clínicos e histopatológicos por microscopia óptica de quatro casos de pacientes com diagnóstico de PCE entre janeiro de 1995 e fevereiro de 2000 no Serviço de Dermatologia da Universidade Federal do Paraná.

Em cada caso, informações clínicas, incluindo sexo, idade, fototipo e ocupação do paciente, foram avaliadas. As características dos tumores, como sintomatologia, curso clínico, tamanho, localização e achados histopatológicos, foram estudadas.

Os tumores de pele surgiram nos pacientes com uma média de 59,2 anos de idade (49-69 anos). Não havia diferenças entre sexos nesse pequeno grupo, totalizando dois homens e duas mulheres. Todos os tumores tinham um crescimento de longa data, de um a 20 anos (média de 7,2 anos), considerando a identificação clínica pelo paciente.

As lesões tinham várias apresentações clínicas: papular, verrucosa, nodular ulcerada e úlcero-vegetante, com tamanhos variando entre $1,2 \times 2$ e $4 \times 5 \mathrm{~cm}$ (Figuras $1 \mathrm{e} \mathrm{2}$ ). Três delas eram localizadas nas extremidades; a outra, na face (Figura 3). Os pacientes apresentavam queixas discretas, como dificuldades ocasionais nos movimentos dos membros, drenagem de líquido claro e sangramento ao trauma. Nenhum deles apresentava linfonodos regionais clinicamente envolvidos.

Dois pacientes tinham carcinoma basocelular (CBC) associado, ambos no nariz. Em um deles, o CBC foi diagnosticado no momento do diagnóstico do PCE (caso 4); e, no outro, uma ressecção prévia de um tumor com exame histopatológico confirmou CBC. O último paciente também tinha hanseníase e foi tratado com poliquimioterapia por dois anos (caso 2).

Todos os pacientes foram submetidos a biópsias, dois deles com achados de PE, sem evidências de

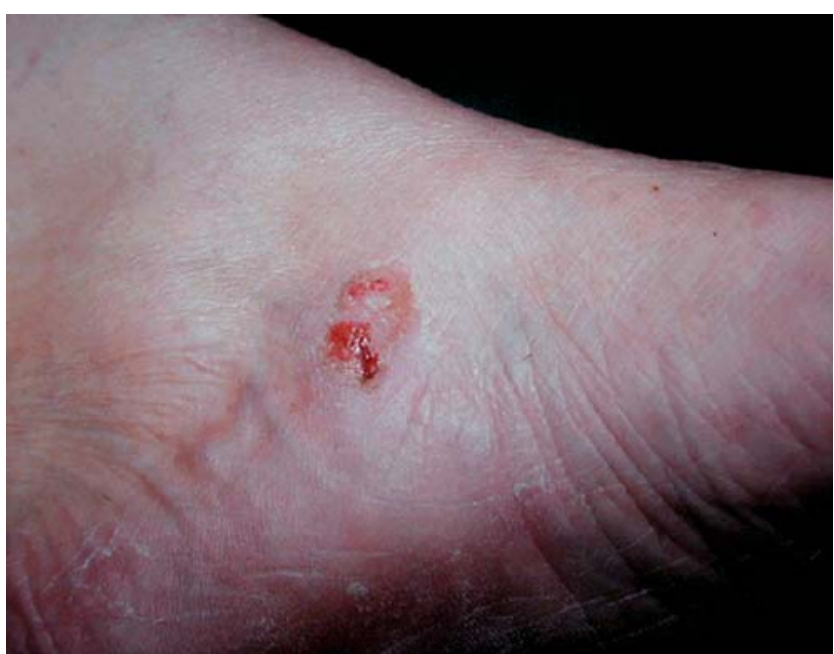

FIgURA 1: (Caso 1) Lesão nodular com ulceração central na borda interna do pé esquerdo; notar a drenagem clara na porção ulcerada

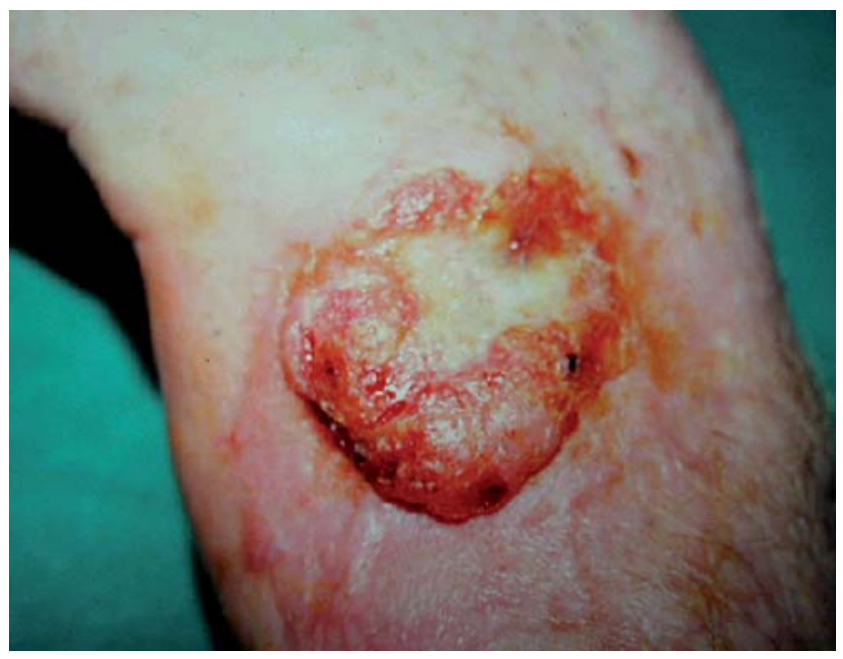

Figura 2: (Caso 2) Lesão úlcero-vegetante no dorso da mão esquerda; pele atrófica circundando a lesão

células atípicas. Após ressecção total do tumor, o estudo histopatológico demonstrou malignidade em todos os casos (Figuras 4 e 5 ).

Uma lesão que teve ressecção inicial incompleta (caso 2) recorreu localmente após dois meses, sendo completamente retirada com nova intervenção cirúrgica. Evidências de metástases não foram encontradas. Todos os pacientes permaneceram livres da doença em um seguimento médio de 7,5 meses (6 a 9 meses). Os resultados estão descritos no quadro 1.

\section{DISCUSSÃO}

O PCE ocorre mais comumente em idosos, com a maioria dos casos na quinta década de vida. Homens e mulheres são igualmente afetados, e a prevalência entre os sexos varia de acordo com os estudos. ${ }^{3.5}$ Aproximadamente 50\% dos PCE surgem nas extremidades inferiores, com mais de $40 \%$ ocorrendo abaixo

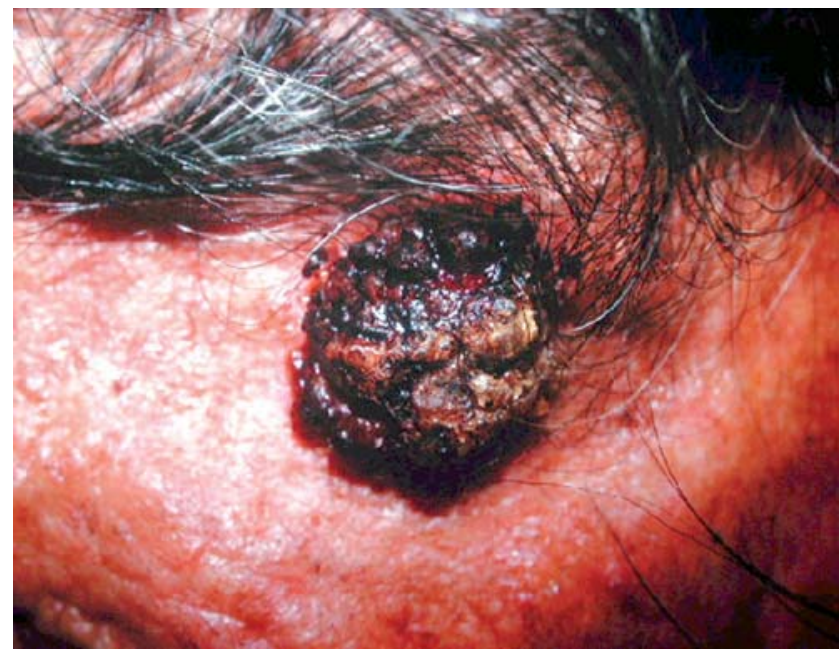

Figura 3: (Caso 4) Lesão verrucosa na região temporal esquerda 


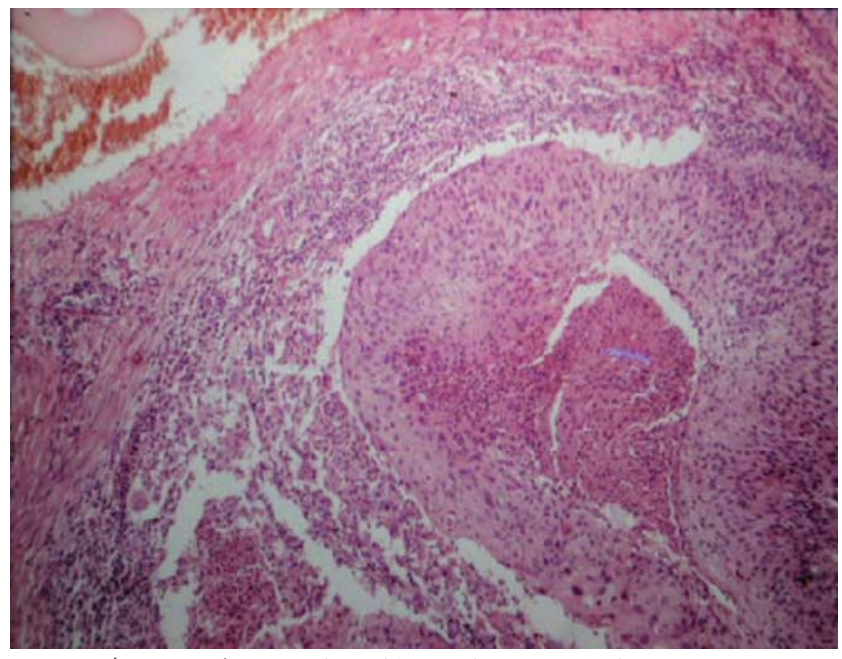

Figura 4: (Caso 4) Corte histológico demonstrando necrose maciça e figuras de mitose no porocarcinoma écrino (HE $100 \mathrm{x}$ )

dos joelhos, ${ }^{2}$ ao contrário dos outros tumores anexiais, que tendem a concentrar-se na cabeça e pescoço. Menos de 5\% ocorrem no couro cabeludo. ${ }^{6}$ Outros sítios afetados são face, ouvido, pálpebras, abdome, vulva, corpo peniano e púbis. Nos casos descritos a idade média quando o tumor foi notado foi de 51 anos. O tempo até o diagnóstico variou de um a 20 anos e a idade média dos pacientes foi de 51 anos. As lesões ocorreram nas extremidades (mão, pé e braço) e na face. Ambos os sexos foram afetados, mas as mulheres (casos 1 e 3) tiveram lesões menores e com curso mais prolongado que os homens.

As lesões geralmente medem menos de $2 \mathrm{~cm}$. Elas se apresentam clinicamente como nódulos solitários circunscritos ou placas na face e extremidades cobertas, de cor rósea ou eritematosa, cobertas por pele normal, podendo ser ulceradas ou com crostas hemáticas. Há descrições de lesões com padrão zosteriforme e múltiplas com distribuição em pedras de calçamento. Muitas são assintomáticas, mas podem ser associadas com sangramento, dor ou prurido.

Os casos relatados ilustram a variedade da apresentação clínica do PCE - desde uma pápula de $1,5 \mathrm{~cm}$ até uma lesão extensa, com áreas ulceradas e bordas vegetantes -, demonstrando a dificuldade do diagnóstico. Sintomas discretos locais foram referidos pelos quatro pacientes.

Há poucas descrições de nevos sebáceos de Jadassohn e hidroacantoma simples mostrando transformação maligna em PCE, mas o papel como possível precursor pré-invasivo dessas lesões ainda não é conhecido. ${ }^{7}$ Parece que a porção superior do ducto écrino dérmico poderia participar na oncogênese do hidroacantoma simples. Mais recentemente, sugeriu-se que uma mutação no gene supressor tumoral, O P53, poderia estar envolvido na carcinogênese do PCE. ${ }^{8}$

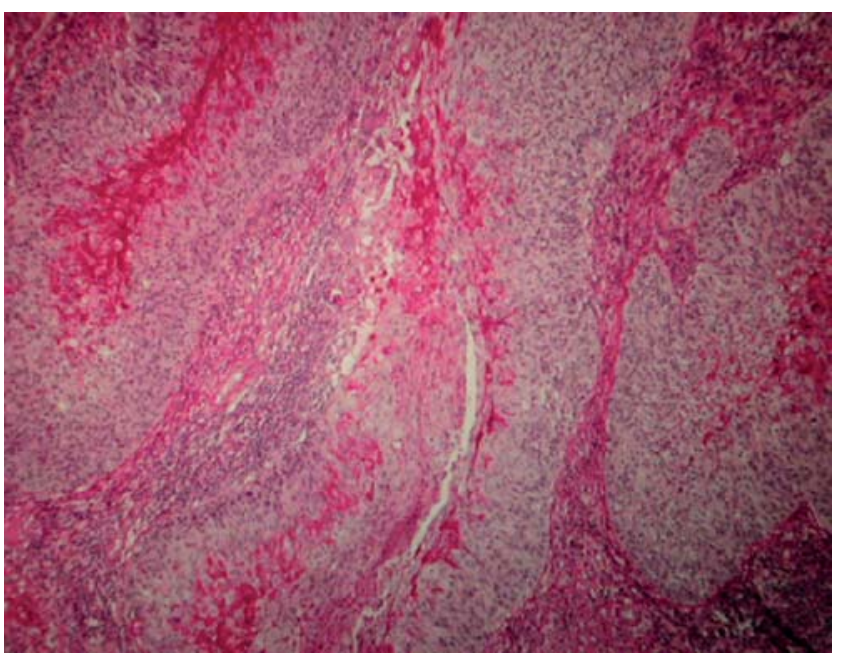

Figura 5: (Caso 4) Corte histológico demonstrando a presença de glicogênio intracelular no porocarcinoma écrino (PAS $400 \mathrm{x}$ )

Muitos pacientes notaram a lesão primária anos antes do diagnóstico. Isso reforça o fato de que muitos PCE podem originar-se de um PE. ' Há também uma descrição demonstrando a relação entre diabetes e PCE, e já estabelecida com o siringoma de células claras. Isso sugere PCE de células claras como marcador cutâneo de diabetes mellitus. ${ }^{10}$ Casos descritos na lâmina e leito ungueal sugerem o trauma como um fator oncogênico. Esta série apresentou dois casos com CBC, o que poderia sugerir uma relação entre PCE e exposição solar. Além disso, todos os pacientes eram do fototipo II ou III, como muitos pacientes da revisão da literatura.

O diagnóstico diferencial inclui carcinomas basocelular e espinocelular, ceratose seborreica, melanoma amelanótico, verruga vulgar, metástase cutânea de neoplasias e outros tumores como doença de Bowen, doença de Paget e hidroacantoma simples. ${ }^{11}$ Em geral, o tumor deve ser considerado em pacientes idosos com tumores de curso prolongado.

O diagnóstico definitivo é histológico, uma vez que os achados clínicos são variáveis. As células neoplásicas são poligonais com citoplasma claro, multinucleadas ou com um núcleo grande, hipercrômico e irregular; estruturas ductais, crescimento pagetoide de células atípicas na epiderme e figuras mitóticas são geralmente observadas. ${ }^{2}$ A epiderme pode apresentar acantose como resultado de numerosos ninhos bem definidos de células tumorais de entremeio.

O diagnóstico histológico pode ser difícil. A presença abundante de grânulos de glicogênio no interior do citoplasma das células tumorais - facilmente observados pela coloração com ácido periódico Shiff (periodic acid Shiff - PAS) -, falta de ceratinização e focos de formação de ductos são características peculiares do PCE que auxiliam no diagnóstico diferencial. ${ }^{12}$ As células do PCE coram-se com o antíge- 
QuADro 1: Dados comparativos dos quatro casos de porocarcinoma écrino

\begin{tabular}{|c|c|c|c|c|}
\hline Achado & Caso 1 & CAso 2 & Caso 3 & Caso 4 \\
\hline Sexo & Feminino & Masculino & Feminino & Masculino \\
\hline Ocupação & Do lar & Porteiro & Do lar & Agricultor \\
\hline Fototipo & II & II & III & III \\
\hline $\begin{array}{l}\text { Idade ao surgimento do } \\
\text { tumor }\end{array}$ & 44 anos & 61 anos & 41 anos & 62 anos \\
\hline Idade ao tratamento & 49 anos & 64 anos & 61 anos & 63 anos \\
\hline Sintomatologia & Drenagem clara & Dor à mobilização & Dor à mobilização & Sangramento \\
\hline Evolução & 5 anos & 3 anos & 20 anos & 1 ano \\
\hline Tipo de lesão & Nodular-ulcerativo & Verrucoso-ulcerativo & Papular & Verrucoso \\
\hline Tamanho & $1,2 \times 2 \mathrm{~cm}$ & $4 \times 5 \mathrm{~cm}$ & $1,5 \times 1,5 \mathrm{~cm}$ & $2,5 \times 3 \mathrm{~cm}$ \\
\hline Localização & $\begin{array}{l}\text { Borda interna do pé } \\
\text { esquerdo }\end{array}$ & $\begin{array}{l}\text { Dorso da mão } \\
\text { esquerda }\end{array}$ & Antebraço esquerdo & $\begin{array}{l}\text { Região temporal } \\
\text { esquerda }\end{array}$ \\
\hline Outras lesões associadas & - & $\begin{array}{l}\text { CBC em face } \\
\text { Hanseníase }\end{array}$ & - & $\mathrm{CBC}$ em face \\
\hline Achados da biópsia inicial & PCE & PCE & $\mathrm{PE}$ & $\mathrm{PE}$ \\
\hline $\begin{array}{l}\text { Margem no tratamento } \\
\text { cirúrgico }\end{array}$ & $0,3 \mathrm{~cm}$ & $0,5 \mathrm{~cm}$ & $0,3 \mathrm{~cm}$ & $0,4 \mathrm{~cm}$ \\
\hline Seguimento & 6 meses & 8 meses & 9 meses & 8 meses \\
\hline
\end{tabular}

PCE: porocarcinoma écrino; PE: poroma écrino; CBC: carcinoma basocelular

no carcinoembrionário (CEA), geralmente encontrado no citoplasma das células tumorais e no lúmen das glândulas malignas. Tais achados permitem a diferenciação entre os tumores de anexo e carcinomas epidérmicos, que são CEA negativos.

O PCE pode desenvolver-se a partir de poroma écrino (PE) intraepidérmico ou dérmico. Nesses casos, podem ser encontradas áreas compostas por células do PE com aparência benigna entre as células anaplásicas. ${ }^{11}$ Logo, é possível que uma amostra pequena obtida por biópsia possa demonstrar características similares às do $\mathrm{PE}$, o que ocorreu em dois dos quatro casos apresentados. Os casos 3 e 4 tiveram biópsias sem indícios de malignidade. Ambos mostraram limites aparentes entre a epiderme e o tumor com células cuboidais PAS positivas. Nenhuma atipia nuclear foi observada nos fragmentos de biópsia. Os quatro casos demonstraram células atípicas PAS posi- tivas e essa coloração foi decisiva para o diagnóstico. Nenhum nicho tumoral foi localizado entre os vasos linfáticos.

Desde 1963, na primeira observação de Pinkus e Mehregan, é bem definido que o estágio inicial do PCE pode ser originado com ilhotas na epiderme. Esse estágio pode ser seguido por envolvimento dérmico. ${ }^{11,12}$ Algumas células do PCE invadem canais linfáticos, que se tornam alargados. Metástases regionais estão presentes em aproximadamente $20 \%$ dos casos. ${ }^{2}$ Metástases cutâneas múltiplas, pápulas ou nódulos, são características peculiares. Metástases viscerais estão raramente presentes no momento do diagnóstico em aproximadamente $12 \%$ dos casos. ${ }^{2,6,12,13} \mathrm{O}$ prognóstico desse carcinoma permanece difícil de ser estabelecido devido à falta de seguimento dos casos descritos na literatura e raridade do tumor. Cerca de $59 \%$ de todos os pacientes apresentam metástases cutâneas, 
viscerais ou de linfonodos regionais ao diagnóstico. ${ }^{14}$ Aproximadamente $20 \%$ dos casos recorrem após excisão, e a mortalidade pode alcançar $67 \%$ em cinco anos se houver envolvimento dos linfonodos regionais.

O tratamento eletivo para o PCE é a excisão cirúrgica total e com amplas margens do tumor. Criocirurgia e eletrocirurgia ainda não apresentam dados de literatura suficientes para avaliar-se precisamente a indicação e a recorrência do tumor. ${ }^{7}$ Radioterapia e quimioterapia parecem ser ineficazes para controlar a recorrência ou metástase tumoral. ${ }^{6}$ Quimioterapia consistindo de metotrexate, cisplatina, adriamicina e bleomicina ou isotretinoína e interferon alfa tem sido usada com respostas parciais. Não há tra-

\section{REFERÊNCIA}

1. Whitt P, Whelchel J, Ruff T. Eccrine Porocarcinoma. Ear Nose Thorat J. 1996:536-8.

2. Berke A, Grant-Kels JM. Eccrine sweat gland disorders: part I - neoplasms. Int J Dermatol. 1994;33:79-85.

3. Poiares Baptista A, Tellechea O, Reis JP, Cunha MF, Figueiredo P. Porocarcinome Eccrine - Revue de 24 cas. Ann Dermatol Venereol. 1993;120:107-15.

4. Ruffieux C, Ramelet AA. Porocarcinoma eccrine. Dermatologica. 1985;170:202-6.

5. Walsh MS. A case of eccrine poroma. J R Soc Med. 1990;83:529-30.

6. Okada N, Ota J, Sato K, Kitano Y. Metastasizing eccrine sweat gland carcinoma. Arch Dermatol. 1984;120:768-9.

7. Turner JJ, Maxwell L, Bursle GA. Eccrine porocarcinoma: a case report with light microscopy and ultraestructure. Pathology. 1982;14:469-75.

8. Akalin T, Sen S, Yuceturk A, Kandiloglu G. P53 protein expression in eccrine poroma and porocarcinoma. Am J Dermatopathol. 2001;23:402-6.

9. Bottles K, Sabegiel RW, Mcnutt NS, Jensen B, Denevey K. Malignant eccrine poroma. Cancer. 1984;53:1579-85.

10. Requeña L, Sarasa JL, Piqué E, Fariña MC, Olivares M, Martín L. Clear-cell porocarcinoma: another cutaneous marker of diabetes mellitus. Am J Dermatopathol. 1997;19:540-4. tamento definitivo disponível para PCE efetivo até o momento. ${ }^{15}$

Todos os casos foram tratados cirurgicamente por excisão ampla com margens de 0,3 a $0,5 \mathrm{~cm}$, que parece ser uma boa opção terapêutica. Houve um caso de recorrência, em cuja biópsia havia comprometimento das margens profundas. Essa recorrência foi local, sem acometimento dos linfonodos regionais após oito meses.

O PCE deve ser lembrado no diagnóstico diferencial em pacientes acima dos cinquenta anos com tumores de curso prolongado nas extremidades e na cabeça. A biópsia é indicada e, mesmo com diagnóstico compatível com PE, deve-se atentar ao risco de malignidade.

11. Elder D, Elenitsas R, Ragsdale BD. Tumors of the epidermal appendages. In: Elder D, Elenitsas R, Jaworsky C, Jonhson B, editors. Lever's Histopathology of the skin. Philadelphia: JB Lippincott; 1997. p.747-803.

12. Mehregan AH, Hashimoto K, Rahbari H. Eccrine adenocarcinoma: a clinopathologic study of 35 cases. Arch Dermatol. 1983;119:104-14.

13. Wick MR, Goellner JR, Wolfe JT, Daniel Su WP. Adnexal carcinomas of the skin. Cancer. 1985;56:1147-62.

14. Matloub HS, Cunningaham MW, Yousif NJ, Sanger JR, Romano JA, Choi HY. Eccrine porocarcinoma. Ann Plast Surg. 1998;20:381-5.

15. Barzi AS, Ruggeri S, Recchia F, Bertoldi I. Malignant metastatic eccrine poroma. Proposal for a new therapeutic protocol. Dermatol Surg. 1997;23:267-72.

ENDEREÇO PARA CORRESPONDÊNCIA / MAILING ADDRESS:

Fabiane Andrade Mulinari-Brenner

Rua General Carneiro, 181

$S A M-4$

80060900 Curitiba PR

Tel.:/fax: 5541 3360-1800 / 2525405

Como citar este artigo/How to cite this article: Mulinari-Brenner FA, Mukai MM, Bastos CAS, Amaral Filho EA, Santamaria JR, Fillus Neto J. Porocarcinoma écrino: relato de quatro casos e revisão da literatura. An Bras Dermatol. 2009;84(5):519-23. 